\title{
INFLUENCIA DEL DISEÑO PERIODÍSTICO EN LA COMPRENSIÓN DE LA INFORMACIÓN
}

\author{
The influence of journalistic design in the \\ understanding of information
}

\author{
Blas-José Subiela-Hernández y Ariana Gómez-Company
}

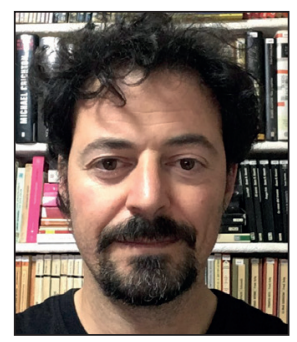

Blas-José Subiela-Hernández es doctor en comunicación por la Universidad Católica San Antonio de Murcia (UCAM) y profesor de diseño en la Facultad de Comunicación de la misma universidad. Es Investigador principal del grupo de investigación Publicidad y RRPP: redefiniciones, públicos y medios y su investigación se centra en las relaciones entre el diseño publicitario y el diseño periodístico a través de la retórica visual. Es autor de varias publicaciones en el ámbito del diseño gráfico desde la perspectiva de la comunicación.

http://orcid.org/0000-0002-7540-9260

bsubiela@ucam.edu

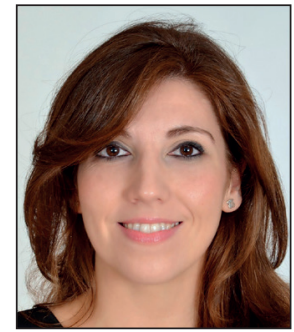

Ariana Gómez-Company es investigadora y doctoranda en el Departamento de Ciencias Sociales y de la Comunicación de la Universidad Católica San Antonio de Murcia (UCAM). Profesora de postgrado en el Título de Experto Universitario en Comunicación Política e Institucional. Licenciada en periodismo y diplomada en estudios avanzados (DEA), formó parte como ayudante de investigación del proyecto Economía de la Participación 20+20 (2013-2014). Sus líneas de investigación son el diseño periodístico y la retórica de la comunicación.

http://orcid.org/0000-0001-9503-4585

ariana.gcompany@gmail.com

Universidad Católica de Murcia Avda. de los Jerónimos, 135. 30107 Guadalupe (Murcia), España

\section{Resumen}

Se plantea un método experimental para examinar las relaciones entre diseño gráfico y comprensión de la información publicada en revistas y/o diarios impresos. Se pretende observar si el diseño gráfico utilizado en un reportaje periodístico hace posible una mejor comprensión del significado de la información. La hipótesis de partida es que el diseño, más allá de garantizar la legibilidad del texto, puede participar en una comprensión del relato más rápida y más eficaz, además de hacerlo más atractivo. Se trata de un estudio con un enfoque multidisciplinar novedoso, ya que no existen investigaciones previas en las que se relacione metodológicamente el diseño periodístico y la comprensión lectora. El objetivo es proporcionar un modelo que ayude a reivindicar el papel del diseño periodístico como una variable para aportar valor añadido tangible al periodismo de calidad, frente a una información cada vez más estandarizada en la que el diseño ha quedado relegado a sus funciones más básicas.

\section{Palabras clave}

Diseño periodístico; Legibilidad; Comprensión lectora; Retórica de la imagen; Caligrama; Prensa impresa; Experimentos; Método experimental.

\begin{abstract}
An experimental method to examine the relationships between graphic design and comprehension of information published in magazines and/or print journals is presented. The method is used to observe if the graphic design in a journalistic report makes it possible to have a better understanding of the meaning of the information. The starting hypothesis is that the design, beyond guaranteeing the readability of the text, can support understanding the story more quickly and effectively, in addition to making it more attractive. This research uses a new multidisciplinary approach, and no known previous research exists in which journalistic design and reading comprehension are methodologically related. The objective of this model is to establish the fundamental role of journalistic design in quality journalism, which runs contrary to the current popularity of basic standardization.
\end{abstract}




\section{Keywords}

Journalistic design; Readability; Reading comprehension; Visual rhetoric; Shape poetry; Printed press; Experimental method; Experiments.

Subiela-Hernández, Blas-José; Gómez-Company, Ariana (2018). "Influencia del diseño periodístico en la comprensión de la información". El profesional de la información, v. 27, n. 1, pp. 115-123.

https://doi.org/10.3145/epi.2018.ene.11

\section{Antecedentes teóricos}

\subsection{Diseño estructural y diseño discursivo}

El diseño periodístico impreso (DPI) tiene la función principal de organizar, jerarquizar y ordenar las diferentes noticias en la página de una forma gráfica y tipográfica (Canga-Larequi, 1994; García, 1998; Lallana, 2000; Zorrilla-Ruiz, 2002; González-Díez; Pérez-Cuadrado, 2001, Gäde, 2002). Se trata de un diseño funcional (Arnold, 1969), que estructura la información para garantizar su legibilidad. La legibilidad de un texto impreso depende en primer lugar de la composición tipográfica, es decir, de la familia de tipos seleccionada, el cuerpo, la longitud de líneas, la interlínea, el tipo de párrafo, etc. (Unger, 2009). Por otra parte, el orden y la jerarquía se obtienen mediante el uso de retículas y el diseño modular (Zorrilla-Ruiz, 2002).

En la presente investigación el diseño que cumple esta función es denominado diseño estructural. Es el que encontraremos de forma generalizada en cualquier página de un diario o revista impresa, así como en sus versiones digitales, adaptado lógicamente al contexto de las pantallas (no se tendrán en cuenta en este trabajo).

Cuando el DPI ha ido más allá de su función estructural ha sido criticado (Lallana, 2000) y se le ha considerado como un elemento característico de la prensa sensacionalista, incompatible con los usos de los diarios más rigurosos del modelo informativo interpretativo (Martínez-Fresneda, 2008; González-Díez et al. 2015).

Sin embargo, consideramos que el diseño periodístico, más allá de su función estética y estructural, puede hacer uso de los elementos de la retórica de la imagen para transmitir parte del relato al que está dando forma, de modo que mejore la comprensión de la información. Barthes (2002) y su discípulo Durand (1982) ya pusieron de manifiesto la capacidad de la imagen para añadir sentido a un mensaje. Aunque estos autores centraron su atención en la retórica de la imagen publicitaria, encontramos defensores contemporáneos del uso de la retórica de la imagen y del diseño en campos más próximos al diseño periodístico. Así, Gamonal-Arroyo (2011) demuestra la eficacia de la creación de discursos visuales basados en operaciones retóricas para el diseño de portadas de libros.

Gamonal-Arroyo y García-García (2015) sugieren la capacidad discursiva del diseño gráfico, denominación que se adopta en este trabajo. Entendemos que el diseño es una construcción retórica con una estructura narrativa que comunica más allá de su expresión, a través de su discurso.
La conexión entre el contenido del relato periodístico y su expresión visual reduce la concepción meramente estilística del diseño. En palabras de Gamonal-Arroyo y García-García (2015, p. 11):

"podemos considerar las piezas del diseño gráfico como un discurso que trasciende y va más allá de las sustancias expresivas que las conforman. Es decir, un diseño es algo más que un conjunto de signos (imágenes y textos) configurados con un orden y un sentido"

Se considera que el diseño gráfico puede ayudar a crear un relato cuya interpretación se produce en un contexto determinado (en la línea de la semiótica pragmática) que va más allá de la literalidad de los signos empleados y que enriquece el relato verbal con un sentido que se superpone a este y que se construye con los elementos propios del diseño (combinación de imágenes, formas, colores y tipografías).

Desde el diseño periodístico se han producido reflexiones sobre el diseño discursivo en la prensa diaria a partir de la perspectiva de la retórica de la imagen (Subiela-Hernández; Gobantes-Bilbao, 2010). Sin embargo, sus implicaciones desde el punto de vista de la comprensión lectora no han sido investigadas.

\subsection{Legibilidad y comprensión lectora}

Estos conceptos son demasiado próximos y su significado suele confundirse con facilidad, por lo que resulta necesario aclarar la postura que se adopta en esta investigación con respecto a los mismos.

\section{Legibilidad y comprensión son concep- tos diferentes}

La mayoría de las investigaciones sobre legibilidad de textos identifican esta cualidad con la comprensión o no diferencian ambas de forma clara (Szigriszt-Pazos, 1993, Alliende-González, 1994, Barrio-Cantalejo; Simón-Lorda, 2003, Gröne, 2009). Por ejemplo Alliende-González (1994, p. 24) define la legibilidad como:

"el conjunto de características de los textos que favorecen o dificultan una comunicación más o menos eficaz entre ellos y los lectores, de acuerdo con la competencia de éstos y con las condiciones en que realizan la lectura".

Sin embargo, legibilidad y comprensión son conceptos diferentes:

- la legibilidad hace referencia a la facilidad para que el lector pueda decodificar el texto; 
- la comprensión se refiere a la asimilación intelectual del contenido del mismo.

Por lo tanto, consideramos que la legibilidad es una condición indispensable para la comprensión (Hilera-González; Fernández-Sanz; Suárez-Morón, 2012), pero no garantiza que dicha comprensión sea alcanzada. Se puede añadir un concepto más, que hace de puente entre legibilidad y comprensión: la lecturabilidad, para la estructura sintáctica del texto (Vived-Conte; Molina-García, 2012). Así:

- la legibilidad está determinada por el aspecto gráfico externo del texto (diseño y composición tipográfica);

- la lecturabilidad está definida por su estructura lingüística interna (complejidad de sus estructuras sintácticas);

- la comprensión sólo se puede lograr sumando a la legibilidad y a la lecturabilidad otros factores externos al texto verbal, vinculados en principio a la formación y experiencia del lector.

En esta investigación se considera que el diseño discursivo puede ser una variable propia del texto no verbal (externa por tanto al texto verbal) que mejore la comprensión lectora. Para ello tendremos en cuenta afirmaciones como la siguiente:

[el diseño] "nos ayuda a aprender información compleja [...], puede hacer lo complicado muy simple y puede ahorrar tiempo y esfuerzo en la transmisión de la información"1 (Coates; Ellison, 2014, p. 20).

En cuanto a la comprensión lectora, es imprescindible citar el trabajo de Van-Dijk como uno de los principales referentes internacionales. El autor afronta en una de sus obras (Van-Dijk, 1990) las particularidades de la comprensión lectora aplicada a las noticias utilizando un método experimental pero sin tener en cuenta las variables del diseño. En ese trabajo evalúa los procesos de producción de noticias y su comprensión a través de un análisis lingüístico y gramatical, de su contenido y forma, así como de las estructuras de estilo y retórica de la noticia.

El aporte novedoso de su investigación es contemplar la noticia "como un tipo de texto o discurso periodístico" para averiguar las "especificidades estructurales del discurso periodístico"; es decir, "por qué y cómo" los relatos periodísticos se diferencian de otros textos (Van-Dijk, 1990, p. 13-14). En el esquema del texto periodístico que propone, cada ítem informativo puede poseer titular, encabezamiento o diferentes tipos de letra, de modo que estas variables desempeñan una función estructural y contribuyen a expresar los principales temas del hecho.

En el campo de la educación, las investigaciones llevadas a cabo sobre comprensión lectora han sido abundantes y han seguido diferentes métodos de investigación.

Desde un enfoque cognitivo del procesamiento de la información, los estudios sobre cómo se comprende coinciden con las teorías lingüísticas sobre el uso del lenguaje y la configuración de un texto (Colomer, 2005, p. 86). Investigadores como Palincsar y Brown (Solé, 2005) se centran en aspectos como la claridad y la coherencia, el conocimiento previo del lector y las estrategias de comprensión y recuerdo.

La comprensión es definida como un proceso en el que "el lector elabora un significado en su interacción con el texto"
(Pérez-Zorrilla, 2005, p. 123) y consta de varios niveles. En la misma línea García-García (1993) hace la siguiente descripción de los niveles o procesos que implica la comprensión lectora:

- Proceso léxico: referido a la decodificación de signos gráficos y letras escritas. La legibilidad del texto (proporcionada por el diseño estructural) es fundamental para que este primer proceso se produzca con éxito.

- Proceso sintáctico: relativo a la estructura de las oraciones. Las clases de palabras y su estructura morfológica, así como el orden de éstas, aportan información sobre su organización sintáctica y favorecen el acceso a su significado.

- Proceso semántico: relativo a la comprensión del significado de las oraciones, es decir, al contenido del texto. Este proceso ayuda a entender las proposiciones e ideas presentes en el texto. Sin embargo, en esta fase no finaliza el proceso de comprensión lectora porque el lector construye el significado no sólo a partir del propio texto, sino de sus conocimientos. Precisamente aquí es posible entender que los elementos del diseño son unidades de información que expresan conceptos apoyándose no sólo en el propio texto verbal, sino también en los conocimientos del lector (procesos retóricos dentro de la semiótica pragmática).

Desde el punto de vista experimental, resulta muy útil la recopilación realizada por Irrazábal y Molinari-Marotto (2005) sobre técnicas para medir la comprensión lectora. En concreto, para la elaboración del cuestionario de este trabajo se parte de las pruebas llevadas a cabo por Elosúa-deJuan y García-García (1993).

\section{El diseño periodístico puede hacer uso de} los elementos de la retórica de la imagen para transmitir parte del relato al que está dando forma, de modo que mejore la comprensión de la información

\section{Material y método}

El método de esta investigación se basa en un diseño cuasi experimental, que nos ayuda a comprobar si existe una relación causa-efecto entre variables (Ruiz-San Román, 2005, p. 236). En este caso las variables son el diseño discursivo ${ }^{2}$ (variable causa) y la comprensión por parte de los lectores (variable efecto). Y la relación que se pretende comprobar es si el diseño discursivo mejora la comprensión lectora.

Aunque el empleo del método experimental es discutido en ciencias sociales (Giddens, 1998, Ritzer, 2000), estamos de acuerdo con Nó-Sánchez y Muñiz-Velázquez (2013) cuando señalan la importancia del uso de estos métodos en la investigación en comunicación.

En concreto, el experimento consiste en mostrar un mismo reportaje periodístico, por la versatilidad de este género y sus posibilidades de puesta en página, con diseño diferente (discursivo y estructural) a dos grupos de individuos (experimental y control) con condiciones socioculturales similares.

El método requiere dividir la muestra en grupo experimen- 
tal y grupo de control porque es la única forma de conseguir el aislamiento necesario. Así, garantizamos que la relación causa-efecto está condicionada exclusivamente por las variables controladas por el estudio (Ruiz-San-Román, 2005) y no se ve influida por otras circunstancias externas.

La variable que diferencia el texto mostrado a un grupo y a otro es, por lo tanto, la del diseño: se muestra al grupo de control el reportaje dotado de diseño estructural (orden, jerarquía y legibilidad), mientras que se facilita al grupo experimental el reportaje dotado de diseño discursivo. En ambos casos se trata de diseño impreso sobre papel.

El diseño discursivo corresponde al publicado en el cuadernillo dominical Crónica del diario El mundo el 20 de marzo de 2016, en sus páginas 10 y 11, bajo el titular "Mi vuelta al mundo en 12 pares de zapatillas" ${ }^{3}$. Se caracteriza por:

- el empleo de un caligrama en el que se agrupan todos los textos (titular, entradilla y cuerpo);

- el silueteo de una imagen para eliminar su fondo;

- la utilización de una caja de forma irregular (que cierra el caligrama) para una fotografía;

- un sencillo esquema.

Por su parte, el diseño estructural se ha construido con el mismo texto verbal a partir de la retícula y los estilos de diseño del diario para este cuadernillo y aplicando los principios básicos del diseño estructural (organización modular y jerarquía). En la construcción del diseño estructural se han utilizado los mismos materiales gráficos que en el diseño discursivo, por lo que la superficie de papel empleada ha sido menor. El texto es un reportaje sobre la vuelta al mundo a pie de Nacho Dean, el primer español en realizar un viaje de este tipo. Ambos diseños se muestran en la figura 1.
El experimento se ha llevado a cabo en laboratorio en el mes de octubre de 2017. Las pruebas han sido simultáneas y, además no hay comunicación entre ambos grupos a lo largo de la prueba. Cada grupo lee el reportaje con el diseño correspondiente y después, por medio de un cuestionario, se evalúa el nivel de comprensión de la información que se ha leído.

El tiempo del que disponen ambos grupos para la lectura del texto se ha establecido a partir de la velocidad media de lectura de un sujeto adulto, que sería de 250 palabras por minuto de acuerdo con la mayoría de los autores (Fry, 1988; Fink, 1992; Buzan, 1998; Olmos-Migueláñez et al., 2011). Dado que el texto del reportaje está formado por 1.329 palabras, el tiempo ofrecido para la lectura es de seis minutos. No obstante, tras el primer minuto de lectura se realizará una pausa para plantear a los lectores una batería de 4 preguntas de supervisión, con las que se pretende medir la comprensión y el interés inicial que despierta el texto. Con estas preguntas se averigua si el diseño discursivo facilita una comprensión más rápida de la información, de acuerdo con uno de los objetivos planteados inicialmente.

El cuestionario se ha elaborado siguiendo las aproximaciones de Elosúa-de-Juan y García-García (1993) sobre las estrategias de comprensión lectora, y se ha tenido en cuenta el diseño de los cuestionarios empleados por Van-Dijk (1990) en sus pruebas experimentales para medir la comprensión de las noticias en dos diarios holandeses.

Las preguntas son de respuestas cerradas y han sido diseñadas para que cubran todos los aspectos de la comprensión lectora. Cuando se ha tratado de identificar una valoración, se ha recurrido a escalas de Likert de 5 posiciones. El orden

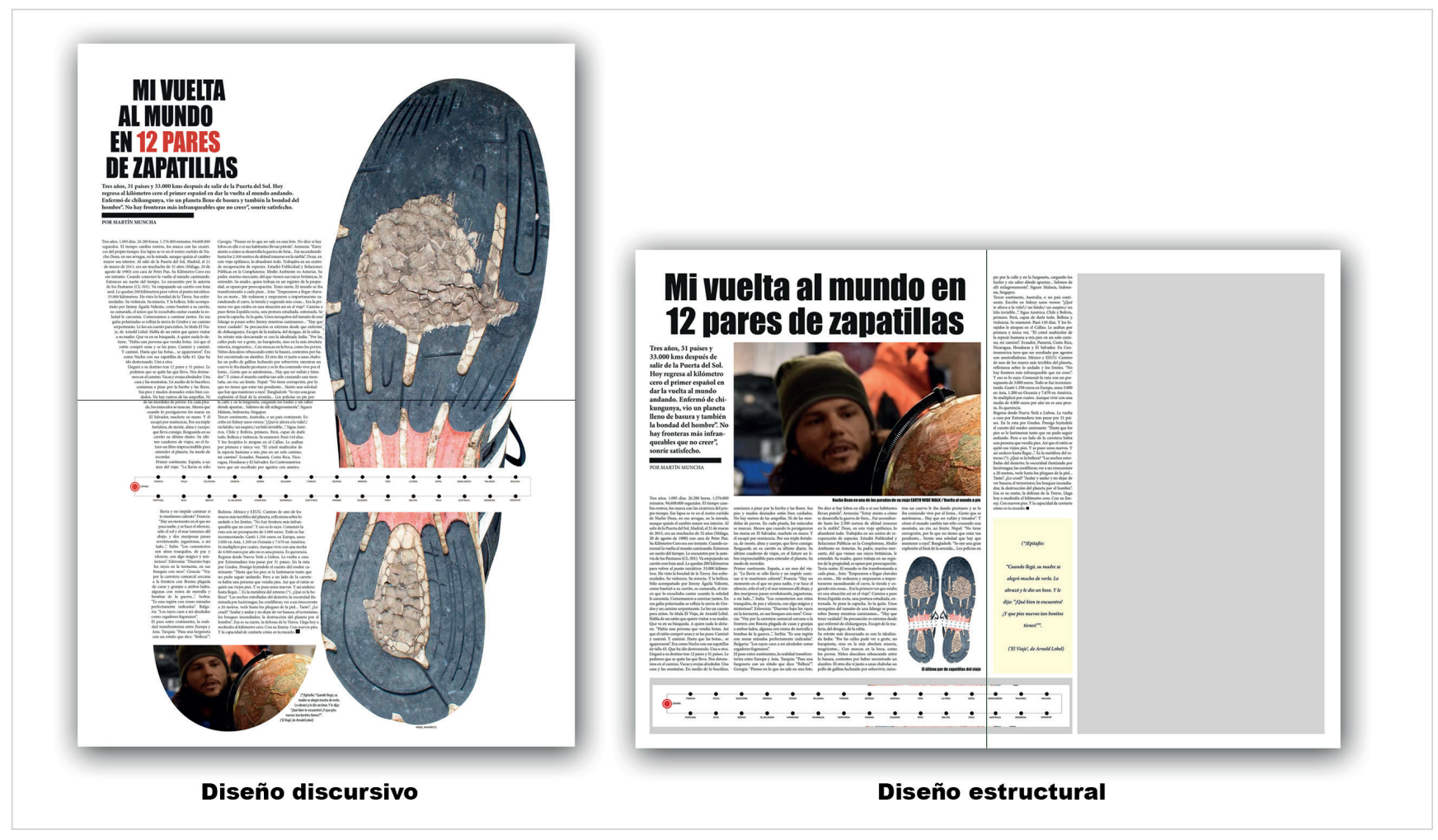

Figura 1 
de distribución de las preguntas es el siguiente:

- segmentación social: edad y sexo;

- preguntas de supervisión contestadas tras el primer minuto de lectura: para valorar la primera impresión del texto desde el punto de vista del interés y la comprensión;

- preguntas sobre el tiempo de lectura: aunque el foco de la investigación no está en el tiempo de lectura, esta es una variable que se ha tenido en cuenta y sobre la que se ha encuestado porque parece clara la conexión entre velocidad y comprensión lectora;

- sobre planificación y estrategias de focalización: para observar si existen conocimientos previos sobre el texto y sobre el plan de lectura;

- sobre estrategias de organización (para determinar si se han identificado las ideas principales del texto),

- sobre estrategias de comprobación y evaluación: referidas a la organización del texto y a las posibles dificultades afrontadas en la lectura;

- sobre el atractivo y el interés que ha despertado la lectura;

- preguntas para definir el nivel de comprensión a través de la identificación de las 5 Ws del periodismo.

Por último, se ha añadido un espacio para que cada individuo pueda aportar su opinión de forma libre sobre la lectura.

Ambos grupos responden al mismo cuestionario. Asumimos que la selección de la muestra sobre la que se aplica el experimento no puede ser aleatoria y no nos posibilita generalizar los resultados obtenidos. De ahí que hablemos de método cuasi experimental y no de diseño experimental, de acuerdo con Wimmer y Dominick (1996, p. 102).

La recogida de datos del experimento se realiza a través de un cuestionario diseñado a partir de la investigación de la comprensión lectora que está influida por variables propias y ajenas al texto (vinculadas con la experiencia y formación del lector); la muestra ha sido seleccionada buscando el máximo nivel de homogeneidad sociocultural de los individuos. De acuerdo con Rossi y Freeman (1989), conseguimos que el grupo de control sea similar al grupo experimental en los aspectos más sustanciales, para minimizar los límites propios de una muestra no aleatoria.

Los participantes en el experimento son estudiantes universitarios españoles de grado (Criminología), tanto varones como mujeres, con edades comprendidas entre 18 y 23 años. En total se trata de 46 lectores divididos en dos grupos homogéneos: 23 lectores para el grupo experimental y 23 lectores para el grupo de control.

Este experimento se debe entender como una prueba exploratoria previa. Por lo tanto, los resultados obtenidos a partir de esta muestra se utilizan fundamentalmente para comprobar la validez del método y para, en su caso, aplicar correcciones al diseño del experimento, pero no se deben considerar concluyentes.

Tras la lectura completa del texto, los lectores del grupo experimental han considerado que es más atractivo que los del grupo de control

\section{Resultados}

Con respecto al primer bloque de preguntas, para valorar si el diseño discursivo facilita una comprensión más rápida de la información, los resultados son muy similares en ambos grupos. No hay diferencias en el nivel de comprensión inicial, aunque con respecto al interés que ha despertado el texto sí se observa que en el grupo experimental ha sido mayor que en el grupo de control (20 individuos frente a 18). Esta tendencia se confirma en el segundo bloque de preguntas, en el que 22 lectores del grupo experimental consideran que ha sido una lectura interesante, frente a 21 del grupo de control. En el gráfico 1 se puede apreciar cómo en las preguntas referidas al interés el grupo experimental supera levemente al grupo de control.

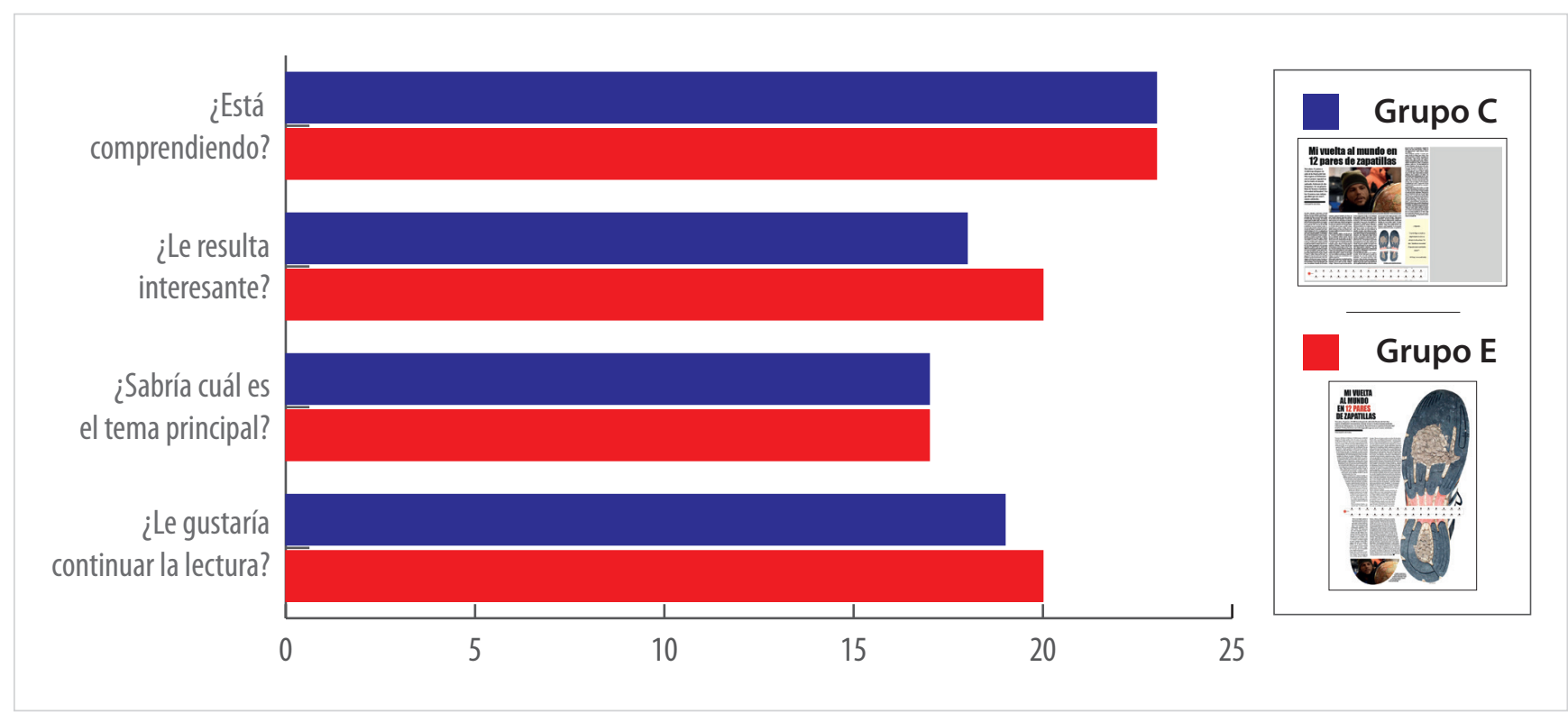

Gráfico 1. Comprensión e interés generados por el texto cuando ha transcurrido un minuto de lectura 


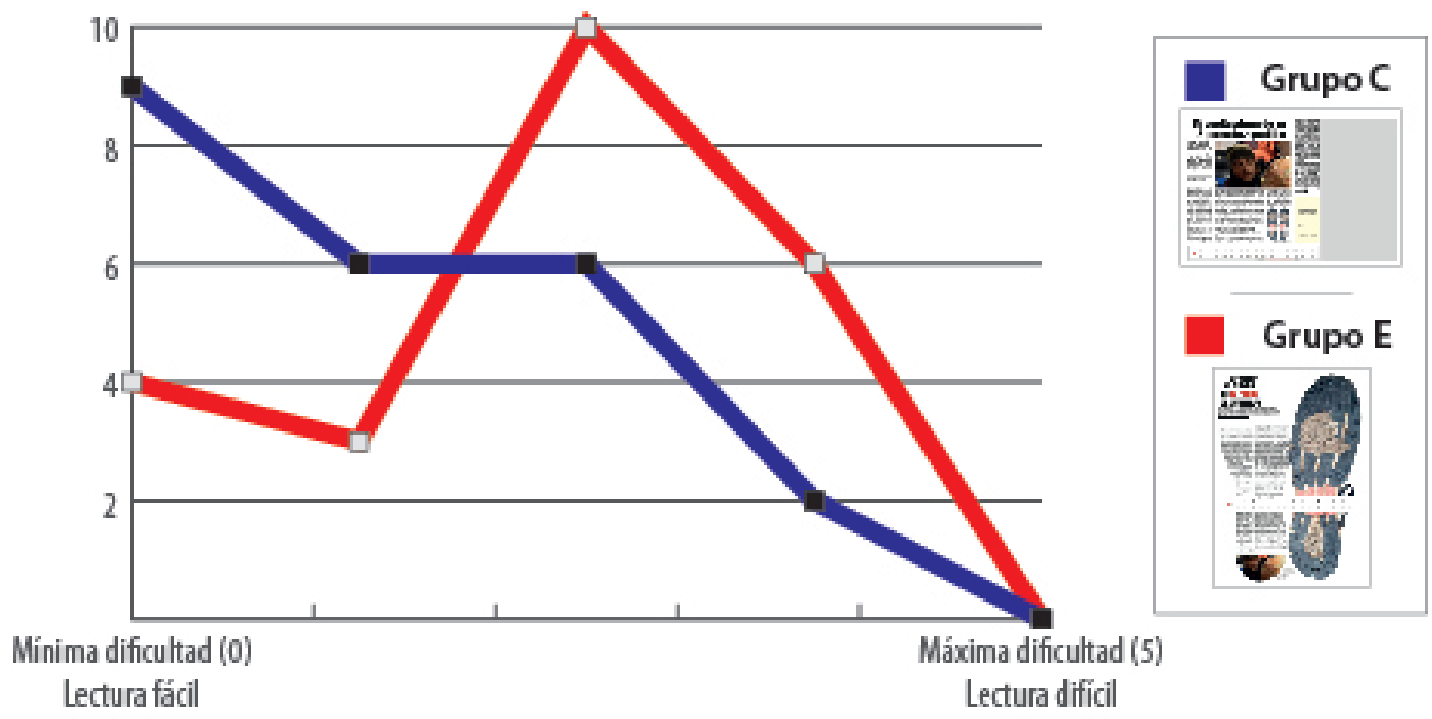

Gráfico 2. La dificultad de lectura ha sido superior para los individuos del grupo experimental

Desde el punto de vista de la velocidad lectora, hay más individuos que han podido leer el texto completo en el grupo de control que en el experimental (10 frente a 8). Además, hay más individuos que han dejado la lectura por la mitad en el grupo experimental (3) que en el de control (1), aunque la mayoría en ambos grupos ha manifestado que ha tenido que detener la lectura en la parte final del texto. En cualquier caso, sorprende que los valores de velocidad lectora sean tan bajos en una población universitaria. $Y$ es un dato que se ve reforzado por los obtenidos en la valoración del nivel de dificultad de la lectura. Es significativo que los individuos del grupo experimental han encontrado, en general, más difícil la lectura que los individuos del grupo de control. Como se puede observar en el gráfico 2, en el grupo de control 9 lectores no han encontrado ninguna dificultad, frente a sólo 4 del grupo experimental, mientras que en este grupo 6 de ellos lo han considerado de dificultad media, frente a sólo 2 con esta valoración en el grupo de control.
Sin embargo, todos los lectores del grupo experimental afirman haber comprendido el tema principal del reportaje, a pesar de la dificultad manifestada en el bloque de preguntas anterior. La diferencia en este aspecto con el grupo de control es poca, puesto que 21 de los 23 individuos del grupo de control indican haber comprendido el tema principal del texto.

En la mayoría de las preguntas relativas a la comprensión profunda del texto (estructuradas en torno a las $5 \mathrm{Ws}$ del periodismo) la ventaja ha sido para el grupo experimental, con diferencias de hasta 20 puntos frente al de control. En concreto, en 6 de las 10 preguntas de este bloque, el grupo experimental ha obtenido mejores resultados que el grupo de control. Sin embargo, en aquellas preguntas cuya respuesta se encuentra al final del texto, la comprensión por parte del grupo experimental ha descendido. Este dato resulta coherente si tenemos en cuenta que los individuos del grupo del control han leído más rápido que los del gru-

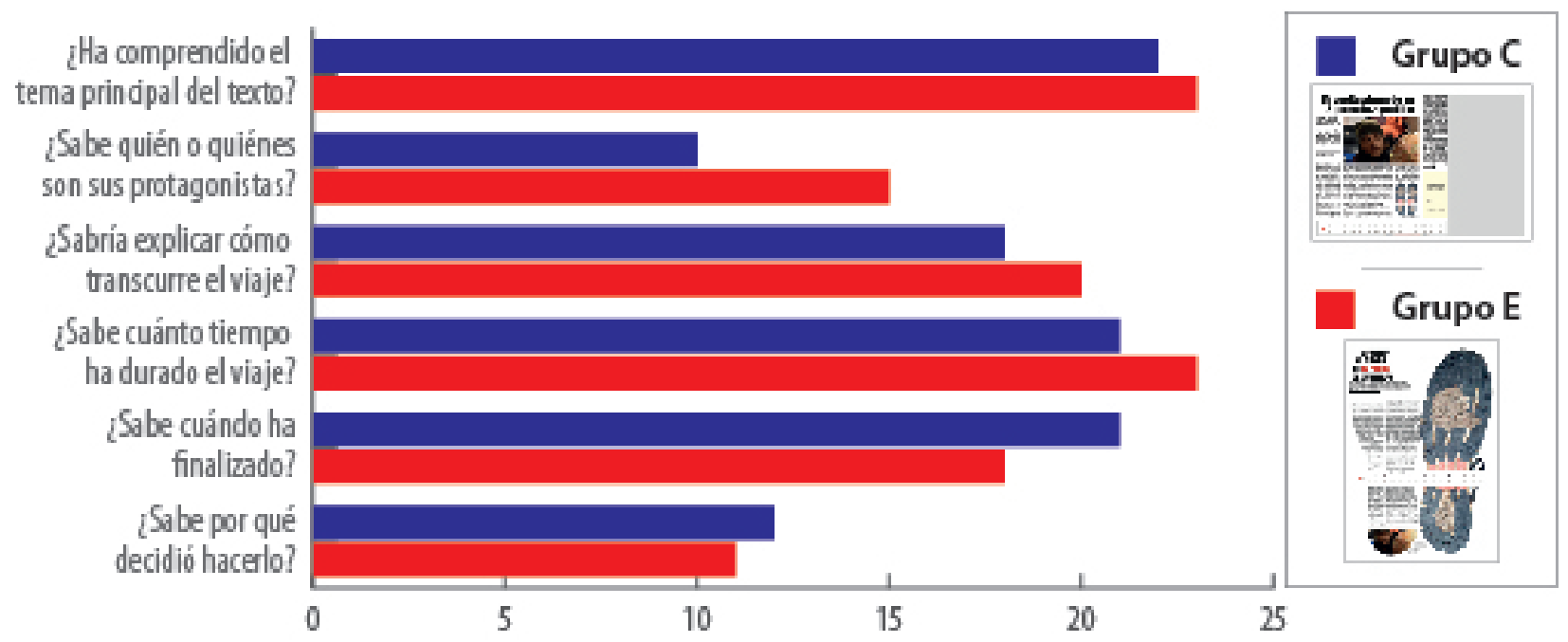


po experimental (todos los lectores del grupo de control han terminado la lectura o la han tenido que abandonar en la parte final, mientras que en el grupo experimental hasta 3 lectores han dejado la lectura por la mitad y sólo 8, como ya se ha indicado, han sido capaces de terminarla). En el gráfico 3 se puede apreciar cómo la comprensión lectora del grupo experimental se reduce a medida que las preguntas hacen referencia a cuestiones que se encuentran al final del texto.

Destacan entre los comentarios libres de los lectores, tres individuos del grupo experimental que consideran que la lectura ha sido difícil por razones "ajenas al texto" y coinciden en que no están acostumbrados a leer con un formato que no consideran habitual (formato "no habitual", "forma irregular" y "párrafos desalineados").

\section{El diseño discursivo no proporciona una} comprensión más rápida de la información ni facilita la lectura, pero sí aporta un conocimiento más profundo del relato periodístico

\section{Conclusiones}

Según los resultados obtenidos en esta prueba, el diseño discursivo no facilita una comprensión más rápida de la información al comienzo de la lectura. Aunque sí hace que el texto resulte más interesante en esos momentos iniciales. Por lo tanto, una de las hipótesis que se planteaba al inicio de la investigación (el diseño discursivo proporciona una comprensión más rápida de la información y hace más atractivo el texto) se ve confirmada sólo en la segunda afirmación. Tras la lectura completa del texto, los lectores del grupo experimental han considerado que es más atractivo que los lectores del grupo de control.

En cuanto a la principal hipótesis del trabajo (el diseño discursivo facilita una mayor comprensión de la información) sí ha sido confirmada, puesto que la comprensión del reportaje tras la lectura ha sido claramente superior en el grupo de lectores que ha utilizado el diseño discursivo. Y ello a pesar de que han considerado la lectura más difícil y han leído más despacio que los individuos del grupo de control. Dos razones podemos apuntar, ambas relacionadas con la novedad del formato experimental:

- como reconocen en sus comentarios tres de los lectores, la distribución del texto en una forma innovadora parece añadir dificultad a la lectura;

- el diseño experimental seleccionado para esta prueba obliga a colocar la doble página en disposición horizontal, frente a la clásica vertical. Al tratarse de una disposición a la que los lectores no están habituados, la dificultad puede derivarse precisamente de esta circunstancia física externa.

Aunque no ha sido afirmado específicamente por ningún lector, podemos entender que el comentario "formato no habitual" puede relacionarse con esta circunstancia. Para el diseño discursivo se ha utilizado el diseño real publicado por El mundo, pero parece necesario optar, para próximas pruebas, por diseños en los que la disposición física de la página no deba ser cambiada entre el grupo de control y el grupo experimental.

En resumen, el diseño discursivo no proporciona una comprensión más rápida de la información ni facilita la lectura, pero sí parece aportar un conocimiento más profundo del relato periodístico. Además influye positivamente en el atractivo y en el interés que despierta el texto.

Más allá de estas conclusiones vinculadas a los resultados obtenidos con el método experimental, hay que proponer los siguientes aspectos para la discusión:

El método ha demostrado su validez, aunque requiere una corrección en cuanto a la preparación de los materiales para que no se produzcan diferencias de orientación física entre los dos diseños.

Para que los resultados sean representativos es necesario reutilizar el experimento propuesto con grupos más numerosos, asegurando su homogeneidad sociocultural, y con materiales más variados (más parejas de diseños diferentes y con variables visuales diferentes, con infografías más detalladas, sin caligramas, etc.) Se recomienda el análisis de los diferentes elementos del DPI de forma individualizada. Con los materiales de este experimento se ha probado especialmente la efectividad de los caligramas como elemento discursivo del diseño. En próximos experimentos se puede poner el foco en la infografía, en la tipografía de los titulares, en el color, en los fondos, etc., de modo que se pueda determinar si existen variables concretas del diseño que mejoran la comprensión.

\section{El diseño discursivo ocupa más espacio} que el diseño estructural, especialmente por el uso del caligrama para la distribución del texto frente a la columna modular

Precisamente la elaboración del material ha puesto en evidencia, al menos para el caso concreto del reportaje de EI mundo utilizado, que el diseño discursivo ocupa más espacio que el diseño estructural, especialmente por el uso del caligrama como forma para la distribución del texto frente a la columna modular. Esto implica que, para igualdad de texto, el diseño estructural podría mostrar más elementos gráficos (fotografías, infografías, despieces) con lo que se cubriría así otra de las demandas de los lectores del grupo de control ("más elementos visuales"). Además, este mismo lector ha encontrado dificultad en la lectura por la extensión del texto. Lo que refuerza la tendencia, ya evidenciada por Suárez-Carballo, Martín-San-Román y Nó-Sánchez (2014), de la hiperfragmentación en el relato de la prensa escrita para hacerlo más accesible.

\section{Notas}

1. Traducción propia del texto original en inglés:

"It helps us to learn complex information [...]. It can make the complicated very simple and can save time and effort in the transmission" 
2. Como ya se ha explicado a lo largo del texto, siguiendo a Gamonal y García-García (Gamonal-Arroyo; García-García, 2015), entendemos que cuando el diseño es capaz de aportar sentido al relato verbal se convierte en discurso que complementa al texto. Frente al diseño estructural, que se limita a ordenar y embellecer, el diseño discursivo aporta sentido al relato.

3. Hemos obtenido autorización de Rodrigo Sánchez, director de arte del diario El mundo, para utilizar y reproducir su diseño en este trabajo.

\section{Referencias}

Alliende-González, Felipe (1994). La legibilidad de los textos: manual para la evaluación, selección y elaboración de textos. Santiago de Chile: Andrés Bello. ISBN: 978956 1312395

Arnold, Edmund (1969). Modern newspaper design. New York: Harper and Row.

Barrio-Cantalejo, Inés-María; Simón-Lorda, Pablo (2003). “¿Pueden leer los pacientes lo que pretendemos que lean? Un análisis de la legibilidad de materiales escritos de educación para la salud". Atención primaria, v. 31, n. 7, pp. 409-414. https://doi.org/10.1016/S0212-6567(03)79199-9

Barthes, Roland (2002). Lo obvio y lo obtuso. Imágenes, gestos, voces. Barcelona: Paidós. ISBN: 9788475094007

https://jpgenrgb.files.wordpress.com/2017/01/barthes-loobvio-y-lo-obtuso-1986.pdf

Buzan, Tony (1998). El libro de la lectura rápida. Barcelona: Ediciones Urano. ISBN: 9788479532222

Canga-Larequi, Jesús (1994). El diseño periodístico en prensa diaria. Barcelona: Bosch. ISBN: 9788476762943

Coates, Kathryn; Ellison, Andy (2014). An introduction to information design. London: Laurence King Publishing. ISBN: 9781780673257

Colomer, Teresa (2005). "La enseñanza y el aprendizaje de la comprensión lectora". En: Lomas, Carlos (comp.). El aprendizaje de la comunicación en las aulas. Barcelona: Paidós Ibérica, pp. 85-105. ISBN: 9878449311772

Durand, Jacques (1982). "Retórica e imagen publicitaria". En: Metz, Christian; Eco, Umberto; Durand, Jacques; Péninou, Georges; Morin, Violette; Du-Pasquier, Sylvain; Fresnaut-Deruelle, Pierre; Bertin, Jacques; Schefer, Jean-Luis. Análisis de las imágenes. Barcelona: Ediciones Buenos Aires. ISBN: 8485989058

Elosúa-de-Juan, Rosa; García-García, Emilio (1993). Estrategias para enseñar y aprender a pensar. Madrid: Ediciones Narcea. ISBN: 9788427710443

Fink, Diana (1992). Técnicas de lectura rápida. Bilbao: Deusto. ISBN: $842340742 \mathrm{X}$

Fry, Edward (1988). Técnica de lectura veloz: manual para el docente. México: Paidós. ISBN: 9688530263

Gäde, Reinhard (2002). Diseño de periódicos. Sistema y método. Barcelona: Gustavo Gili. ISBN: 9788425218491
Gamonal-Arroyo, Roberto (2011). "Retórica aplicada a la enseñanza del diseño gráfico". Icono14, v. 9, n. 3, pp. 410-422. https://doi.org/10.7195/ri14.v9i3.128

Gamonal-Arroyo, Roberto; García-García, Francisco (2015). "La capacidad discursiva del diseño gráfico". Arte, individuo y sociedad, v. 27, n. 1, pp. 9-24.

https://doi.org/10.5209/rev_ARIS.2015.v27.n1.43009

García-García, Emilio (1993). "La comprensión de textos. Modelo de procesamiento y estrategia de mejora". Didáctica, v. 5, pp. 87-113.

https://revistas.ucm.es/index.php/DIDA/article/viewFile/ DIDA9393110087A/20216

García, Mario (1998). "Tendencias en comunicación periodística visual". Revista latina de comunicación social, n. 3. https://www.ull.es/publicaciones/latina/z8/marz98.mario. htm

Giddens, Anthony (1998). Sociología. Madrid: Alianza. ISBN: 9788420684673

http://www.perio.un/p.edu.ar/catedras/system/files/ anthony_giddens_-_sociologia.pdf

González-Díez, Laura; Pérez-Cuadrado, Pedro (2001). Principios básicos sobre diseño periodístico. Madrid: Universitas. ISBN: 9788479911157

González-Díez, Laura; Puebla-Martínez, Belén; Birkner, Thomas; Pérez-Cuadrado, Pedro (2015). "El diseño periodístico como actor fundamental en los modelos de prensa popular. Una aproximación a las causas del cierre del diario Claro". Revista latina de comunicación social, n. 70, pp. 859-877. https://doi.org/10.4185/RLCS-2015-1075

Gröne, Oana (2009). "Inventario de instrumentos para medir la legibilidad de un texto en castellano". Health literacy. https://goo.gl/4PnYw2

Hilera-González, José-Ramón; Fernández-Sanz, Luis; Suárez-Morón, Esther (2012). “Evaluación de la legibilidad de webs de universidades". El profesional de la información, v. 21 , n. 5 , pp. 468-474.

https://doi.org/10.3145/epi.2012.sep.04

Irrazábal, Natalia; Molinari-Marotto, Carlos (2005). “Técnicas experimentales en la investigación de la comprensión del lenguaje". Revista latinoamericana de psicología, v. 37, n. 3, pp. 581-594

http://www.redalyc.org/articulo.oa? $?$ id $=80537309$

Lallana, Fernando (2000). Tipografía y diseño. Madrid: Síntesis. ISBN: 9788477387411

Martínez-Fresneda, Humberto-José (2008). "El diseño periodístico, clave en la lectura crítica de la prensa". Comunicar, v. 15 , n. 30. pp. 179-184.

https://doi.org/10.3916/c30-2008-02-012

Nó-Sánchez, Javier; Muñiz-Velázquez, José-Antonio (2013). "El estado de la metodología experimental en la investigación en Comunicación". En: Vicente-Mariño, Miguel; González-Hortigüela, Tecla; Pacheco-Rueda, Marta. Investigar la comunicación hoy. Revisión de políticas cientificas y aportaciones metodológicas. Simposio internacional sobre política 
científica en comunicación. 2 o Congreso nacional sobre metodología de la investigación en comunicación, pp. 123-130. ISBN: 9788461641246

https://dialnet.unirioja.es/servlet/articulo?codigo $=4227308$

Olmos-Migueláñez, Susana; Borham-Puyal, Miriam; García-Riaza, Blanca; Rodríguez-Conde, María-José; Elorza, Izaskun (2011) "Velocidad y comprensión lectora: planificación de la carga académica en la universidad". Bordón, v. 63, n. 3, pp. 107-119.

https://dialnet.unirioja.es/descarga/articulo/3712075.pdf

Pérez-Zorrilla, María-Jesús (2005). "Evaluación de la comprensión lectora: dificultades y limitaciones". Revista de educación, n. extraordinario, pp. 121-138.

https://goo.gl/tbM7PJ

Ritzer, George (2000). Teoría sociológica clásica. Madrid: MacGraw-Hill. ISBN: 8448101790

https://significanteotro.files.wordpress.com/201

Rossi, Peter; Freeman, Howard (1989). Evaluación. Un enfoque sistemático para programas sociales. México, D.F.: Trillas.

Ruiz-San-Román, José-Antonio (2005). "Los experimentos". En: Berganza-Conde, María-Rosa; Ruiz-San-Román, José-Antonio (coords.) Investigar en comunicación. Madrid: MacGraw-Hill. ISBN: 9788448198251

Solé, Isabel (2005). "De la lectura al aprendizaje". En: Lomas, Carlos (comp.). El aprendizaje de la comunicación en las aulas, pp. 107-122. Barcelona: Paidós Ibérica. ISBN: 84 49311772

Suárez-Carballo, Fernando; Martín-San-Román, Juan-Ra- món; Nó-Sánchez, Javier (2014). “La hiperfragmentación informativa en el diseño periodístico impreso: propuesta metodológica y análisis de repercusiones cognitivas". Icono14, v. 12 , n. 1, pp. 63-104.

https://doi.org/10.7195/ri14.v12i1.627

Subiela-Hernández, Blas-José; Gobantes-Bilbao, Maite (2010). "La retórica visual en el diseño periodístico: un diagnóstico". En: Il Jornadas de diseño en la sociedad de la información, pp. 31-39. ISBN: 9788469533895

https://dialnet.unirioja.es/descarga/articulo/4165956.pdf

Szigriszt-Pazos, Francisco (1993). Sistemas predictivos de legibilidad del mensaje escrito: fórmula de perspicuidad. Tesis doctoral. Universidad Complutense de Madrid.

http://biblioteca.ucm.es/tesis/19911996/S/3/S3019601.pdf

Unger, Gerard (2009). ¿Qué ocurre mientras lees? Tipografía y legibilidad. Valencia: Campgràfic. ISBN: 9788496657120

Van-Dijk, Teun (1990). La noticia como discurso. Comprensión, estructura y producción de la información. Barcelona: Paidós Comunicación. ISBN: 8575096220 https://goo.gl/KgiK7t

Vived-Conte, Elías; Molina-García, Santiago (2012). Lectura fácil y comprensión lectora en personas con discapacidad intelectual. Universidad de Zaragoza. ISBN: 9788415538783

Wimmer, Roger; Dominick, Joseph (1996). La investigación científica de los medios de comunicación. Una introducción a sus métodos. 1a Edición. Barcelona: Bosch. ISBN: 84 $7676359 x$

Zorrilla-Ruiz, Jesús (2002). Introducción al diseño periodístico. Pamplona: Eunsa. ISBN: 9788431320324
La Fundación Biblioteca Social es una institución sin ánimo de lucro que se constituyó en el año 2014. Tiene como objetivo contribuir a compensar los desequilibrios sociales apoyando proyectos que llevan a cabo las bibliotecas públicas, dirigidos a los sectores más vulnerables de la sociedad.

\section{¿Colaboras?}

fundacionbibliotecasocial.org info@fundacionbibliotecasocial.org

3 @Biblio_Social

FundacionBibliotecasSocial

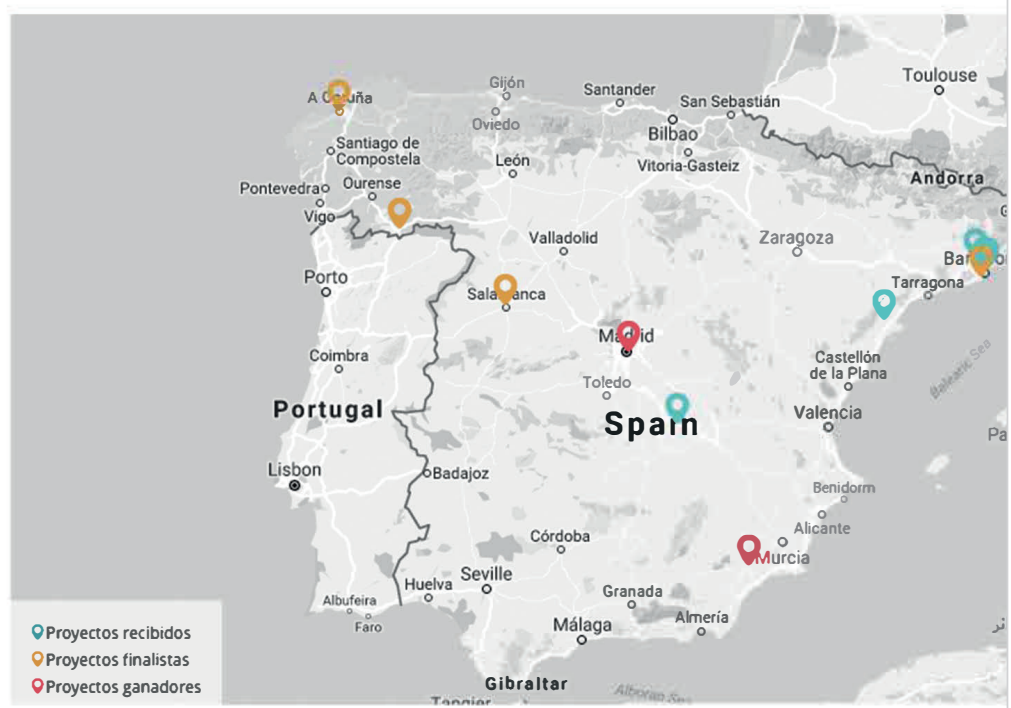

Mapa de proyectos de bibliotecas públicas para la inclusión social. 2016.

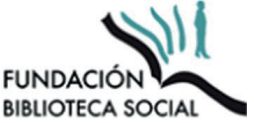

